\title{
Optics program in the physics department at San Jose State University
}

Ramendra Bahuguna, Karamjeet Arya, Joseph Becker, John Gruber, H. Sarma Lakkaraju, et al.

Ramendra Deo Bahuguna, Karamjeet Arya, Joseph F. Becker, John B. Gruber, H. Sarma Lakkaraju, Kenneth B. Wharton, Gareth T. Williams, "Optics program in the physics department at San Jose State University," Proc. SPIE 4588, Seventh International Conference on Education and Training in Optics and Photonics, (28 May 2002); doi: 10.1117/12.468715

Event: Education and Training in Optics and Photonics 2001, 2001, Singapore, Singapore 


\title{
The Optics Program in the Physics Department at San Jose State University
}

\author{
Ramen D. Bahuguna, Karamjeet Arya, Joseph F. Becker, \\ John B. Gruber, H. Sarma Lakkaraju, Kenneth B. Wharton, and Gareth Williams \\ Department of Physics, San Jose State University, San Jose, CA 95192-0106, USA
}

\begin{abstract}
The San Jose State University Physics Department, located in Silicon Valley, provides students with a high quality education in optics and provides local high-tech industry and government laboratories with a partner for optics-related research and development projects. There are approximately 50 undergraduate majors and 20 graduate (M.S.) students in the Department. Core courses leading to the B.S. in Physics are offered with upper division courses in Modern Optics, Lasers and Applications, Advanced Optics Lab, Advanced Instrumentation Lab, and Individual Studies as well as graduate courses in Electro-optics, Graduate Optics, Optical Metrology, and Laser Spectroscopy. Graduates are well prepared to enter the lasers and optics industry or go on to graduate school. A 4000 square-foot lab in the Science Building houses the Institute for Modern Optics, an organized research unit in the College of Science. One of the major goals of the Institute is to facilitate collaborative research between the local optics industry and the faculty and students at SJSU. The Department is presently developing a new biophotonics lab for single molecule studies with a dual beam optical tweezers already operational. A National Science Foundation Research Experience for Undergraduates Program grant provides research support for undergraduates.
\end{abstract}

Keywords: optics, lasers, education, research

\section{INTRODUCTION}

San Jose State University, the Metropolitan University of Silicon Valley, is a modern 26,000-student comprehensive university. It is one of the larger campuses of the California State University. New faculty members are hired based on their research interests and capabilities as well as their teaching ability, and are expected to carry out state-of-the-art research projects and excel in teaching. The Department of Physics has a tenure-track faculty of 17 and a student enrollment of 55 undergraduate majors and 20 graduate students (MS candidates). Situated in the heart of high-tech "Silicon Valley," the Department made an early decision to focus in the areas of lasers and optics, condensed matter, and computational physics. Our undergraduate program in Lasers and Optics has received national recognition by the Optical Society of America and our Physics Club has received national recognition and several awards from the American Physical Society.

\section{BS IN PHYSICS WITH AN OPTION IN LASERS AND OPTICS}

The courses in condensed matter and computational physics are very well established and have earned the respect of the local scientific community in Silicon Valley and beyond. In a typical semester there are approximately 20 undergraduates doing independent study research projects in optics, condensed matter and computational physics, and about a half dozen graduate (M.S.) candidates working on their theses. The Physics Department faculty at SJSU, partly as a result of our geographic location, has a unique combination of interests and capabilities in these fields. We are thus in a unique position to offer quality research experiences for undergraduates in the area of optics, condensed matter, and computational physics. 
Students in the Department have an option in Lasers and Optics which consists of a minimum of 12 units of lecture and laboratory courses beyond the B.S. core courses selected from the following:

Advanced Physics Laboratory: Instrumentation

Modern Optics

Advanced Physics Laboratory: Optics

Lasers and Applications

Advanced Physics Laboratory: Lasers, and

Graduate courses in Electro-optics, Optics, Optical Metrology, Laser Spectroscopy, and Individual Study.

\section{INSTITUE FOR MODERN OPTICS}

The Institute for Modern Optics is an organized research unit in the College of Science at SJSU. The Institute was established to facilitate research in optics among faculty members and students in the College. There are presently more than a dozen full-time faculty associated with the Institute, most of whom are physicists. A wide variety of on-going funded research projects is carried out in the Institute using a full complement of lasers and optics-related equipment.

Some of our faculty research is done in our 4,000 square-foot "College of Science Institute for Modern Optics" in the Science Building. The Institute has a variety of lasers, optical tables, spectrometers, interferometers, and the usual complement of oscilloscopes, meters, power supplies, etc. Other faculty research labs are also located in the Science Building, along with a professionally-staffed machine shop, and a professionally-staffed electronics shop. Available equipment used to support student research activities include: Tunable Diode Laser Spectrometer, Raman Spectrometer, Cahn balance, signal conditioning equipment (Lock-in amplifiers, pre-amps, etc.), data acquisition and analysis systems (Personal Computers with A/D and D/A), various lasers (Argon, YAG, HeNe, etc.), Zygo and other interferometers, UV-VIS-IR spectrophotometers, Genessee optical design software, optical tables and components.

\section{PARTNER ORGANIZATIONS}

In addition to our on-campus facilities, off-campus research facilities in industrial and government laboratories provide students with the perspective of a non-academic research laboratory to complement their classroom work.

\subsection{NASA Ames Research Center}

Four projects are carried out in laboratories at NASA Ames Research Center, located about 10 miles from the San Jose State campus, in collaboration with NASA scientists. The nature of the projects range from atmospheric physics to space science to astronomy. The optics related project is directed by Dr. Joseph F. Becker, and involves the development of a stable isotope laser spectrometer for planetary exploration. This work has resulted in the award of two patents and the involvement of a San Diego start-up company SpiraMed, Inc. whose goal is to develop and market a medical instrument based on innovations of Dr. Becker and his NASA collaborator, Dr. Todd B. Sauke. The medical version of the instrument can be used to perform diode laser-based real-time measurements of stable isotopic ratios of carbon in the breath of patients to diagnose various maladies of human internal organs. This effort represents a transfer of technology from the laboratory to the commercial sector.

\subsection{Department of the Army}

One of our optics related projects is directed by SJSU Physics Professor John B. Gruber at SJSU and at the Night Vision and Electronic Sensors Directorate, US Army, CECOM (NVESD) in collaboration with Dr. J. Andrew Hutchinson. Dr. Gruber's project is a collaboration among government, academia, and industry (Aculight, Inc.) which has been successful in transferring technology from the research lab to the commercial sector. 


\subsection{Aculight, Inc.}

SJSU Physics Professor Gruber's NSF-REU project is a collaboration among government (US Army Night Vision Lab), academia, and industry which has been successful in transferring technology from the research lab to the commercial sector.

\subsection{Advanced Precision Technology, Inc.}

SJSU Physics Professor Ramen Bahuguna has been awarded two patents for his work with Advanced Precision Technology, Inc. on holographic-based fingerprint sensors. This work has resulted in an instrument that is being introduced to the commercial market and represents a transfer of technology from the lab to the commercial sector.

\subsection{Lawrence Livermore National Laboratory}

Lawrence Livermore National Laboratory is not far from our campus and is engaged in a variety of scientific investigations. Dr. H. Sarma Lakkaraju and Dr. Kenneth B. Wharton both have collaborations at the National Lab and do their research on campus as well as at Livermore. They bring their students to the lab for research activities during the summer and the academic year.

\section{BREAK-THROUGH DESIGN OF NEW MONOBLOCK LASER}

The project directed by Professor John B. Gruber at San Jose State University and at the Night Vision and Electronic Sensors Directorate, US Army, CECOM (NVESD) in collaboration with Dr. J. Andrew Hutchinson has produced an important result. Dr. Gruber and his students have been active participants in the mission to explore novel materials which will serve as passive Q-switches in laser transmitters for the US Army rangefinder systems. The work has ranged from the spectroscopic analysis of candidate materials, to the modeling and simulation of the new monoblock laser, a break-through design with much promise. The project is a collaboration among government, academia, and industry which has been successful in transferring technology from the research lab to the commercial sector.

Project Description: 'Modeling of Saturable Q-switch Absorbers, Laser Cavity Designs and OPO Materials' Passive Q-switching of miniature lasers operating at 1.33 or 1.54 microns is attractive because the method is simple, compact and requires a less complicated cavity design. If frequency shift is required, selection of efficient robust OPO materials is necessary in order to meet expanding industrial and military expectations. We have successfully modeled the Q-switched Cr4+:NdYAG laser with an OPO providing output frequencies at 1.33 and 1.54 microns. This modeling carried out by numbers of our team over the past three years include students S. Amad, A. Kennedy, and S. Maples. The results have led the U.S. Army through its Chemical-Electronics Command to reduce size and improve efficiency of eye-safe laser range finders, and remote sensing devices.

The Q-switch model makes use of coupled rate equations based on four-level or three-level gain media and a fourlevel absorber medium including the effects of excited-state absorption. Numerical integration techniques are used to develop solutions as analytical functions prove cumbersome to describe the dynamics of the cavity system. Modeling of OPO materials such as KTP and KAP, including novel borate crystals are currently underway. Measurement of refractive indices, infrared transmission and modeling of power loss and efficiencies are planned. Several local small businesses, The Army Research Laboratory, and the Naval Research Laboratory have all expressed interest in these design/modifications to current state-of-the art technology in near infrared lasers as illuminators as well as range finders. Selected publications ${ }^{1-4}$ related to monoblock laser developments are listed in the Reference section. 


\section{SINGLE MOLECULE STUDIES OF BIOMOLECULAR PROCESSES}

The tremendous growth in the field of molecular biology during the past two or three decades has opened new avenues of basic and applied research for physicists. A significant outcome of this growth for example is the human genome project. ${ }^{5}$

Biological and biophysical studies at the molecular level can, of course, be carried out with aggregates of molecules and the bulk of our understanding to date has been deduced in this fashion. The inherent limitation of such measurements is that one obtains an average of a large number events, which are perhaps identical or perhaps not. On the other hand, single molecule measurements remove this averaging. If one were to perform a large number of single molecule measurements, one can generate a distribution of measured values which contains much more information than a simple average value. Single molecule measurements are one class of the state-of-the-art experimental methods in molecular biology and biophysics today. Three different techniques are in vogue at present, namely confocal fluorescence microscopy/spectroscopy, ${ }^{6}$ laser tweezers ${ }^{7,8}$ and atomic force microscopy. ${ }^{9}$ Another technique that is gaining attention is Surface Enhanced Raman Spectroscopy (SERS). ${ }^{10}$ These techniques are not in lieu of each other but are complimentary to each other.

Professor H. S. Lakkaraju has begun to develop a biophotonics lab for single molecule studies at SJSU beginning with the first generation single beam optical tweezers; since a dual beam optical tweezers has been built. ${ }^{11}$ A Raman spectrometer is also under development at SJSU, which is basically operational but for the data acquisition/processing part. The plan is to complete building the Raman spectrometer and evaluate the sensitivity and performance of the tweezers and the Raman spectrometer first. After this phase, two single molecule techniques will be applied to the measurement of biomolecular processes/systems. These include ultimate sensitivity of immunoassays, DNA repair enzymes (i.e., mechanisms used to find damaged regions and their ability to interact with other enzymes to affect the repair), determination of the sequence of a single DNA fragment using specially labeled polymerase enzymes, and molecular processes at and across cell surfaces.

While the science is rather sophisticated in its content, an undergraduate student can learn and become experienced in modern optical techniques applied to biophysical problems. The students will get training in setting up optics experiments, data reduction and analysis. They will also experience the significance of optical techniques in biophysics. Recently, we established collaboration with Medical Technology Program (MTP) of Lawrence Livermore National Laboratory (LLNL). LLNL also has comprehensive research programs in biosciences and biotechnology having collaborative components with MTP. The interaction the students will have with leading scientists in the field at LLNL will be a valuable asset in their careers and may encourage them to choose biophotonics as their professional path. The SJSU campus already provides excellent education/research opportunities in biotechnology/biological sciences that can be used to strengthening this new interdisciplinary.

\section{NATIONAL SCIENCE FOUNDATION-RESEARCH EXPERIENCE FOR UNDERGRADUATES}

The program's major goal is to involve undergraduate students, especially women and under-represented minorities, in research who might not otherwise have the opportunity. It is generally recognized that active research experience promotes a high quality educational experience for Physics majors and is effective in attracting undergraduates to careers in science. The advancement of scientific progress in the United States requires a well-trained diversified workforce pursuing careers in technical research. The intended impact of the proposed program is to increase the supply of well-trained and experienced researchers to satisfy the demand of the many research and development enterprises in the nation, especially here in high-tech Silicon Valley. The REU site in the Physics Department at San Jose State University is ongoing and has been funded by NSF since 1989.

\section{OPTICS RELATED RESEARCH PROJECTS}

\section{1 "Tunable Diode Laser Spectroscopy of 12C/13C Ratio in Carbon Dioxide" Dr. Joseph F.}




\section{Becker}

The overall objective of this on-going project is to develop a tunable IR semiconductor diode laser gas analysis technique to measure gas concentrations and isotope ratios of various gases in the laboratory and in situ on Mars. From experience with our initial multiple beam $\mathrm{Pb}$-salt diode laser spectrometer which is designed to emit in the 4.3 _ region $\left(2300 \mathrm{~cm}^{-1}\right)$, we have been able to design and test a single compact beam instrument concept with rapid gas handling capabilities and accuracy of better than $0.10 \%$. Tunable diode laser linewidths of approximately $0.0003 \mathrm{~cm}^{-1}$ allow detection of individual rotational lines, and at such high resolution problems of impurity gasses interfering with the measurement can be eliminated. This represents a major advance over the accepted mass spectrometer technique for measuring gas concentrations and isotope ratios (1). Students initially acquire spectroscopic data in our lab at NASA Ames, transfer it to our laboratory computer, and acquire an understanding of the experimental apparatus and theory. As competence rapidly increases, computer analysis of data is performed, including plots of experimental data and theoretical models for comparison. More advanced students make error analyses, suggest improvements in experimental protocols, and align optical components and build and modify the electronics.

\section{2 "Modeling of Saturable Q-switch Absorbers, Laser Cavity Designs and OPO Materials" Dr. John B. Gruber}

Passive Q-switching of miniature lasers operating at 1.33 um or $1.54 \mathrm{um}$ is attractive because the method is simple, compact and requires a less complicated cavity design. If frequency shift is required, selection of efficient robust OPO materials is necessary in order to meet expanding industrial and military expectations.

We have successfully modeled the Q-switched $\mathrm{Cr}^{4+}: \mathrm{NdYAG}$ laser with an OPO providing output frequencies at 1.33 um and $1.54 \mathrm{um}$. The results have led the U.S. Army through its Chemical-Electronics Command to reduce size and improve efficiency of eye-safe laser range finders, and remote sensing devices.

The Q-switch model makes use of coupled rate equations based on four-level or three-level gain media and a fourlevel absorber medium including the effects of excited-state absorption. Numerical integration techniques are used to develop solutions as analytical functions prove cumbersome to describe the dynamics of the cavity system. Modeling of OPO materials such as KTP and KAP, including novel borate crystals are currently underway. Measurement of refractive indices, infrared transmission and modeling of power loss and efficiencies are planned for the coming three-year cycle. Several local small businesses, The Army Research Laboratory, and the Naval Research Laboratory have all expressed interest in the work that Prof. Gruber and his NSF-supported students are doing in these design/modifications to current state-of-the art technology in near infrared lasers as illuminators as well as range finders.

The project has been expanded to include characterization and modeling of novel scintillator materials and rare earthdoped III-V semiconductors. Interpretation of the optical and electrical properties of these materials will result in the participation of the students in presentations at national and international meetings, referred publications, and job opportunities with companies with which we presently hold SBIR and STTR contracts. ${ }^{13}$

The undergraduate students assist in solving the simultaneous rate equations using spectroscopic parameters from potentially useful materials. They will assist in developing new programs and in modifying existing ones in order to improve efficiency and formatting of results. They will be presenting their work at meetings and in invited seminars.

\section{3 "Holographic Fingerprint Sensors" Dr. R. D. Bahuguna}

We have recently developed a holographic fingerprint sensor which corrects the geometric distortion and aberrations present in conventional prism sensors. Several patents and publications are listed in the Reference section. ${ }^{14-19}$ The holographic sensors were made using 8E75 Agfa holographic plates. We now plan to make these sensors using Dichromated gelatin, thereby increasing the diffraction efficiency of the holograms. Our previous research on Dichromated gelatin holograms with REU students will be very useful in this application. 


\section{4 "Modes of Protein Motion at Membrane Surface via Laser Tweezers" Dr. H.S. Lakkaraju}

The tremendous growth in the field of molecular biology during the past two decades has opened new avenues of study for physicists. There is a need to develop tools to monitor and models to understand biological processes at the molecular level. Need for advances in nanotechnology, arguably the next generation technology, provides further impetus for molecular level understanding of physical processes and sensors. With this as a backdrop, we began to build an experimental molecular biophysics program about a year ago here at SJSU. Our laboratory now has an optical tweezers system and several spectroscopic tools including nonlinear spectroscopic techniques. We also have access to an atomic force microscope and an electron microscope. Our primary focus is physics at and across membrane surfaces. Future plans include the following: Since there is no reliable and accurate procedure to compute radiation forces under arbitrary conditions, calibration of the laser tweezers is important. We will do calibration and evaluation of our optical tweezers using polystyrene spheres. We plan to measure the radial and axial stiffness of our tweezers with sphere radius and laser power as parameters. After this preliminary work, we plan to undertake experiments where membrane proteins coated with latex or gold particles are dragged on a membrane surface and the dynamics of particle motion are determined. The objective of such experiments is to characterize and understand the details of modes of motion of proteins. We plan to supplement the single particle motion measurements with second harmonic generation and sum frequency generation measurements that provide information regarding molecular dynamics at surfaces. ${ }^{20}$

The experiments outlined above cover a broad range of degree of difficulty in experimental modern optics/biophysics. Thus undergraduate students will learn how to set up typical modern optics experiments, analyze the data and interpret the information as well. This project will provide an exciting opportunity for an undergraduate student to experience the experimental diversity in a modern optics laboratory at SJSU and gain hands on training in experimental biophysics.

\section{5 "Effect of Interface Roughness on the Electrical and Optical Properties of Low- dimensional Semiconductor Materials" Dr. Karamjeet Arya}

The interface roughness present in low dimension semiconductor materials (quantum wells, quantum wires, etc.) plays an important role in determining their electrical and optical properties. Recently, in collaboration with the experimental group of Prof. Peter Yu, Physics Department, UC Berkeley, We have been working on the phenomenon of photoluminescence up-conversion in semiconductor hetero-structure materials. We have made preliminary calculations for the up-conversion efficiencies using Auger recombination and two-step two-photon absorption mechanism. However, it is found that hetero-structure interfaces in the experiments are not smooth but have some island structures along with small-scale roughness. This roughness is believed to play an important role in the up-conversion efficiency. We now plan to include the effect of this roughness in our calculations. The smallscale roughness will be modeled by a Gaussian function described by root-mean square roughness amplitude and a correlation length. The effect of this roughness will be calculated using the lowest order perturbation theory. The large-scale roughness will then be treated numerically by solving the integral equation, which includes higher order roughness terms. We already have obtained the analytical expressions for the integral equation, which includes the effects of large-scale roughness. These studies will include both analytical and numerical calculations. One or two undergraduate students who have taken their upper division E\&M course and have some computational knowledge will be involved in the project. In addition to gaining research experience, these students will have the opportunities to interact with the experimental research group at UC Berkeley. Hopefully this will motivate them to enter the physics graduate program. ${ }^{21}$

\section{6 "Probing Warm Dense Matter with Ultrafast Lasers" Dr. Ken Wharton}

Warm Dense Matter (WDM) is a regime where the thermal energy of a material is comparable to the ionization energies, making first-principle calculations of material properties extremely difficult. Predictions of the equation of state, electrical conductivity, and expected ionization levels are unreliable in this regime and vary substantially between different simulations. Experimental data is also very sparse, not because such states of matter are difficult to create in the laboratory, but because WDM is a fleeting, transitional state between classical solids and classical plasmas. With the recent advent of ultrafast (<100fs) lasers, it is now possible to probe material properties on a 
sufficiently fast timescale to study WDM (typically at solid density and $0.5-10 \mathrm{eV}$ temperature). Undergraduate students assist with the experiments (carried out in collaboration with Lawrence Livermore National Laboratory), and will learn to postprocess the data into a format which is comparable to simulations. This research will help to benchmark and refine computational material models and will provide needed data in fields ranging from astrophysics to prepulse effects in laser-solid interactions. ${ }^{22}$

\section{ACKNOWLEDGEMENTS}

The following sources have provided funding to support, in part, the research described in this report: National Science Foundation-Research Experience for Undergraduates Program, Night Vision and Electronic Sensors Directorate of US Army, NASA, and San Jose State University Foundation.

\section{REFERENCES}

1. J. B. Gruber with A. Kennedy, and M. Bowers, "Modeling the 1.5 um Monoblock Transmitter for the Microlaser Rangerfinder," Proc. MDAS, Vol. 25, 19227, 1999.

2. J. B. Gruber with A. Kennedy, B. Zandi, and J. A. Hutchinson, "Modeling of the Co2+ Saturable Absorber QSwitch for the E:Yb: Glass Laser (1.53 um)," Proc. SPIE, Vol. 3928, 142, 2000.

3. J. B. Gruber with A. Kennedy, and M. Bowers, "Modeling the 1.5 um Long Pulse Er:Yb:Glass Laser," Proc. MDAS, Vol. 26, 1421, 2000.

4. )," J. B. Gruber with B. Zandi, L. D. Merkle, and J. A. Hutchinson, "Pump-probe Measurements and Modeling of U4+:CaF2 as a Q-Switch for the Er:Yb:Glass Laser (1.53 um"), Technical Digest, CLEO, May 2001.

5. Special Issue on Human Genome, Science 291, 1304, 2001.

6. S. Weiss, "Fluorescence Spectroscopy of Single Biomolecules," Science, 283, 1676, 1999.

7. K. Sveboda, S. M. Block, "Biological Applications of Optical Forces," Annual Rev. Biophys. Biomol. Struct., 23, 247, 1994.

8. A.D. Mehta, et.al., "Single Molecule Biomechanics with Optical Methods" Science, 283, 1689, 1999.

9. J.K. Gimzewski and C. Joachim, "Nanoscale Science of Single Molecules Using Local Probes," Science 283, 1676, 1999.

10. K. Kneipp, et.al., "Single Molecule Detection Using Surface-Enhanced Raman Scattering (SERS,)" Phys. Rev. Letters, 78, 1667, 1997.

11. D.R. Balsley, "Design and Demonstration of Dual Beam Optical Tweezers," Master's Thesis (SJSU), March, 2001.

12. T.B. Sauke and J.F. Becker, "Stable Isotope Laser Spectrometer for Exploration of Mars," Planetary and Space Science, 46, 805-812, 1989; and U.S. Patent No. 5,543,621 awarded to J.F. Becker, et al. (August 6, 1996):

"Laser Diode Spectrometer for Analyzing the Ratio of Isotopic Species in a Substance."

13. J.B. Gruber with B. Zandi, D. Anker, S. Ahmad, and J.A. Hutchinson, "Numerical Modeling of Passive Qswitch Absorbers for Erbium Phosphate Glass Lasers," Proc. MDAS 21, 421, 1997.

14. R.D._Bahuguna and K. Khajehnouri, "An Improved Processing Technique for Dichromated Gelatin Holograms," Industrial Applications of Optical Inspection Metrology and Sensing, Proc. SPIE Vol. 1821, 232, 1992.

15. R. D. Bahuguna and Tom Corboline "Method for Recording a Holographic Optical Element," U. S. Patent No. 6,038,043 March 14, 2000.

16. Tom Corboline and R. D. Bahuguna, "Real Time Fingerprint Sensor and Verification System," U. S. Patent No. 6,002,499 December 14, 1999.

17. R. D. Bahuguna, “Miniature Fingerprint Sensor using a Trapezoidal Prism and a Holographic Optical Element," U.S. Patent No. 5,892,599 April 6, 1999.

18. R. D. Bahuguna and Tom Corboline, "Prism Fingerprint Sensor using a Holographic Optical Element," U. S. Patent No. 5,629,764 May 13, 1997. 
19. J. Esparza, R. D. Bahuguna and G. T. Williams, "Infrared Aided Method and Apparatus for Venous Examination,” U. S. Patent No. 5,608,210 March 4, 1997.

20. A. Ashkin, J.M. Dziedzic, and T. Yamane, "Optical Trapping and Manipulation of Single Cells using Infrared Laser Traps,” Nature, 3330, 769-771, 1987.

21. D.L. Tripp and K. Arya, "Applications of New Variational Principle to Calculate the Electronic States for Low Dimensional Semiconductor Quantum Well Systems," 1995 APS March Meeting, San Jose, CA.

22. K .B. Wharton, C. Boley, A. M. Komashko, A. M. Rubenchik, J. Zweiback, J. Crane, G. Hays, T. E. Cowan, and T. Ditmire, "Effects of Non-Ionizing Prepulses in High Intensity Laser-Solid Interactions," Physical Review E, 64, 025401(R) 2001. 